\title{
EPISODIOS DE TOXICIDAD EN MOLUSCOS DE AGUAS MARINAS COSTERAS DE LA PROVINCIA DE BUENOS AIRES (ARgENTINA) ASOCIADOS A ALGAS TOXÍGENAS (MARZO DE 2008-MARZO DE 2013)
}

\author{
INÉS SUNESEN ${ }^{1,4}$, ANDREA S. LAVIGNE ${ }^{1,2}$, ALEJANDRA GOYA ${ }^{3} y$ \\ EUGENIA A. SAR ${ }^{1,4}$
}

\begin{abstract}
Summary: Toxicity episodes in mollusks from marine coastal waters of the Province of Buenos Aires (Argentina) associated to toxigenic algae (March 2008-March 2013). Since a project of monitoring toxigenic microalgae and toxins in shellfish started in 2008, carried out along the coast between San Clemente del Tuyú and Mar Azul (Northern Zone) and Bahía Anegada (Southern Zone) of the Province of Buenos Aires, four outbreaks of toxicity for paralytic shellfish toxins (PSP) and eight outbreaks for lipophilic shellfish toxins (DSP/not determined) were recorded. The results were used as technical tool from which the implementing authority (Dirección Provincial de Pesca of the Ministerio de Asuntos Agrarios of the Provincia de Buenos Aires) founded eleven decisions to impose closures and one sanitary alert to the consumption, extraction and commercialization of shellfish. Three of the PSP outbreaks occurred in the Northern Zone associated with Gymnodinium catenatum. The fourth episode occurred in Puerto Quequén associated with Alexandrium tamarense. In the same area there were three DSP toxicity events, and associated species were Dinophysis acuminata and D. caudata. The remaining five episodes of toxicity for lipophilic toxins occurred in the Southern Zone, in one or more of the three sanitary areas classified as A by SENASA, and none of them could be associated with any of the known toxigenic species.
\end{abstract}

Key words: Alexandrium tamarense, Dinophysis acuminata, Dinophysis caudata, Gymnodinium catenatum, DSP toxins, lipophilic toxins, PSP toxins.

Resumen: A partir de un monitoreo de microalgas toxígenas y toxinas en moluscos iniciado en 2008, llevado a cabo entre San Clemente del Tuyú y Mar Azul (Zona Norte) y en la Bahía Anegada (Zona Sur) de la Provincia de Buenos Aires fueron registrados cuatro brotes de toxicidad por toxina paralizante de moluscos (PSP) y ocho brotes por toxinas lipofílicas de moluscos (DSP/no determinadas). Los resultados obtenidos fueron empleados como herramienta técnica a partir de la cual la autoridad de aplicación (Dirección Provincial de Pesca del Ministerio de Asuntos Agrarios de la Provincia de Buenos Aires) fundó la decisión de imponer once vedas y una alerta sanitaria al consumo, extracción y comercialización de moluscos. Tres de los brotes por PSP se produjeron en la Zona Norte y la especie asociada fue Gymnodinium catenatum. El cuarto episodio se produjo en Puerto Quequén, durante un muestreo eventual, asociado a Alexandrium tamarense. En esta misma zona se produjeron tres de los eventos de toxicidad por DSP, y las especies asociadas fueron Dinophysis acuminata y $D$. caudata. Los restantes cinco episodios de toxicidad por toxinas lipofílicas ocurrieron en la Zona Sur, en una o más de las tres zonas clasificadas sanitariamente como A por SENASA, y ninguno pudo ser asociado a alguna de las especies toxígenas conocidas.

Palabras clave: Alexandrium tamarense, Dinophysis acuminata, Dinophysis caudata, Gymnodinium catenatum, toxinas DSP, toxinas lipofílicas, toxinas PSP.

\footnotetext{
${ }^{1}$ División Ficología "Dr. Sebastián A. Guarrera", Facultad de Ciencias Naturales y Museo, UNLP, Paseo del Bosque s/n, 1900 La Plata, Argentina. * isunesen@fcnym.unlp.edu.ar

${ }^{2}$ Dirección Provincial de Pesca, Ministerio de Asuntos Agrarios de la Provincia de Buenos Aires, Calle 51 esquina 12, Torre 1, piso 7, 1900 La Plata, Argentina.

${ }^{3}$ Departamento de Toxinas Marinas, Laboratorio Regional Mar del Plata, Centro Regional Buenos Aires Sur, SENASA, Aviso Dorrego y Víctimas del 46, 7600, Mar del Plata, Argentina

${ }^{4}$ CONICET
} 


\section{INTRODUCCIÓN}

Varias especies de diatomeas y dinoflagelados son capaces de producir proliferaciones masivas, relativamente repentinas y espacialmente restringidas de una o unas pocas especies. A estos fenómenos se los conoce como floraciones (blooms), mareas rojas, discoloraciones o alocoloraciones. En cambio, se denomina "floraciones algales nocivas (FAN)" "harmful algal blooms (HAB)" a aquellos eventos que producen niveles de toxinas en moluscos que ponen en riesgo la salud humana, aún con concentraciones celulares moderadas que no llegan a provocar discoloraciones (Reguera, 2002).

Las toxinas de mayor relevancia para la salud pública producidas por microalgas son: toxina paralizante de moluscos ( $\mathrm{PSP}^{1}$ Paralytic Shellfish Poisoning), toxina diarreica de moluscos (DSP Diarrhetic Shellfish Poisoning), toxina amnésica de moluscos (ASP Amnesic Shellfish Poisoning), toxina neurotóxica de moluscos (NSP Neurotoxic Shellfish Poisoning) y toxina ciguatérica (CTX Ciguateric Toxin). Todas ellas pueden causar intoxicaciones alimentarias en el hombre, provocando cuadros de variable gravedad. Los organismos que funcionan como vectores más comunes para que las toxinas producidas por algas lleguen a través de la red trófica al hombre son moluscos filtradores en las cuatro primeras y peces en la última. Estos organismos tienen la capacidad de bioacumular toxinas a partir de las algas que les sirven como alimento.

Tiempo después del primer evento en Argentina de intoxicación por consumo de moluscos contaminados con PSP asociado a la presencia de Alexandrium tamarense (Lebour) Balech ${ }^{2}$, que produjo la muerte de dos pescadores en aguas de la plataforma frente a Península Valdés (Carreto et al., 1981), fueron registrados varios episodios de toxicidad por PSP en moluscos en la Provincia de Buenos Aires, asociados a la presencia de $A$. tamarense y Gymnodinium catenatum Graham. A. tamarense ha producido eventos de toxicidad recurrentes durante la primavera-verano con una

\footnotetext{
1 Los acrónimos utilizados para las toxinas y para el síndrome que ellas producen son los anglosajones dada la diversidad de acrónimos que se ha generado en español.

${ }^{2}$ Carreto et al. (1981) mencionaron la especie bajo el nombre Gonyaulax excavata (Braarud) Balech. Esta fue posteriormente transferida al género Alexandrium Halim bajo el nombre de $A$. excavatum (Braarud) Balech et Tanguen y sinonimizada por Balech (1995) con Alexandrium tamarense (Lebour) Balech.
}

amplia variación interanual de las concentraciones de toxinas (Carreto et al., 1996, 1998 a y b) y $G$. catenatum ha provocado eventos de toxicidad recurrentes durante el otoño (Carreto \& Akselman, 1996; Akselman et al., 1998). Más recientemente Negri et al. 2004) asociaron Pseudo-nitzschia australis Frenguelli a la presencia de toxinas ASP (ácido domoico) en el plancton, carne de mejillón y contenido estomacal de anchoítas durante invierno del año 2000.

A partir del año 2008 fue iniciado un proyecto de "Monitoreo de microalgas de ambientes costeros de la Provincia de Buenos Aires y monitoreo de toxinas en moluscos bivalvos" con la participación conjunta del Ministerio de Asuntos Agrarios, el Servicio Nacional de Sanidad y Calidad Agroalimentaria (SENASA) y la División Ficología "Dr. Sebastián A. Guarrera" de la Facultad de Ciencias Naturales y Museo (UNLP), cuya finalidad es detectar tempranamente especies toxígenas y toxinas en moluscos y transferir la información a la autoridad de aplicación a fin de que la use como herramienta técnica para la toma fundada de decisiones relacionadas con la protección de la salud pública.

El objetivo de este trabajo es presentar los resultados obtenidos sobre especies toxígenas y toxicidad en moluscos de ambientes costeros de la Provincia de Buenos Aires durante el período marzo de 2008-marzo de 2013 en el marco del proyecto de monitoreo citado, y las decisiones de la Dirección Provincial de Pesca a que dichos resultados dieron lugar.

\section{MATERIAL Y MÉTOdOS}

Los muestreos de fitoplancton y moluscos se realizaron quincenalmente entre septiembre y abril y mensualmente entre mayo y agosto, en dos áreas costeras de la Provincia de Buenos Aires:

1) la Zona Marítima Norte, que abarca desde San Clemente del Tuyú hasta Mar Azul (Fig. 1), caracterizada por ser un área turística con extensas playas arenosas, donde suelen extraerse moluscos bivalvos filtradores con propósito de consumo doméstico (extracción no comercial). En caso de bioensayos positivos de los moluscos muestreados en cualquiera de las estaciones, la veda se dispone para la zona completa.

2) la Zona Marítima Sur que comprende la Bahía Anegada (Fig. 1), reconocida a través de la Resolución $\mathrm{N}^{\circ}$ 121/06 de SENASA como primera zona "clase A" productora de moluscos bivalvos en 


\section{Inés Sunesen et al. - Episodios de toxicidad en moluscos asociados a algas toxígenas}

el país, en la que se llevan a cabo actividades de extracción y cultivo de ostra con la consiguiente generación de empleo en el área. Esta zona fue dividida en tres sub-zonas AR-BA 001 (Los Pocitos), AR-BA 002 (Ría del Jabalí) y AR-BA 003 (Bahía San Blas) por Resolución 43/05 del Ministerio de Asuntos Agrarios de la Provincia de Buenos Aires. En caso de bioensayos positivos de los moluscos muestreados la veda se dispone sólo para la sub-zona de donde éstos proceden.

En el año 2009 se iniciaron muestreos conjuntos de la Dirección Provincial de Pesca y del Organismo Provincial de Desarrollo Sustentable de la Provincia de Buenos Aires extendiendo el área muestreada hasta Monte Hermoso, esfuerzo de muestreo que no pudo ser sostenido en el tiempo.

Para el estudio de fitoplancton, fueron tomadas muestras cualitativas por arrastre de red de $30 \mu \mathrm{m}$ de apertura de poro y muestras cuantitativas con botella. Estas fueron fijadas con formalina al $4 \%$ y al $0,4 \%$ respectivamente y una alícuota de la muestra cualitativa fue trasladada en heladera al laboratorio para su observación in vivo. Las muestras cualitativas fueron analizadas con microscopio óptico (MO) con contraste de fase y contraste diferencial de interferencia (DIC) Leica DM 2500 y microscopio electrónico de barrido (MEB) Jeol JSM 6360 LV y las cuantitativas con microscopio invertido Zeiss Axiovert 40 CFL según técnica Utermöhl para estimar el número de células por litro.

Para el monitoreo de toxinas, fueron tomadas simultáneamente muestras de los moluscos a los que se pudo acceder en el área cercana al punto de recolección del fitoplancton. En la Zona Norte de la Provincia se colectó preferentemente almeja (Mesodesma mactroides Deshayes) o berberecho (Donax hanleyanus Philippi) que son especies apetecidas por lugareños y turistas, y para el caso en que no se pudieron localizar bancos de estas especies se muestreó mejillín (Brachidontes rodriguezii d'Orbigny) que comúnmente no es consumido pero resulta útil a los fines del monitoreo por ser un bivalvo con alta tasa de filtración. En la Zona Sur se colectó ostra del Pacífico (Crassostrea gigas Thunberg). Las muestras fueron enviadas en frío para su análisis a laboratorios del SENASA.

Los análisis para detectar toxina paralizante en moluscos (PSP) fueron realizados mediante bioensayo en ratón de acuerdo con la metodología planteada por la AOAC (1995) en ambas zonas desde el comienzo del monitoreo.

Para la determinación de toxinas lipofílicas en moluscos se aplicó la técnica de bioensayo en ratón siguiendo la metodología descripta por Yasumoto et al. (1984) modificado por Yasumoto en Fernández et al. (2002) que permite determinar, en forma conjunta ácido okadaico (AO), dinofisistoxinas (DTXs), pectenotoxinas (PTXs), yesotoxinas (YTXs) y azaspirácidos (AZAs) sobre el cuerpo entero de los moluscos. Los análisis fueron realizados desde el comienzo del monitoreo en la Zona Sur y a partir de enero de 2010 en la Zona Norte luego de detectar especies toxígenas en concentraciones que según Reguera (2002) son consideradas críticas. Aquellas muestras positivas para toxinas lipofílicas fueron posteriormente analizadas en la mayoría de los casos con cromatografía líquida de alta resolución con detección por fluorescencia (HPLC-FLD) siguiendo el método descripto por García et al. (2010).

Los análisis para detectar toxina amnésica de moluscos (ASP) fueron realizados sólo para muestras procedentes de la Zona Sur, y la técnica utilizada fue la de cromatografía líquida de alta resolución (HPLC).

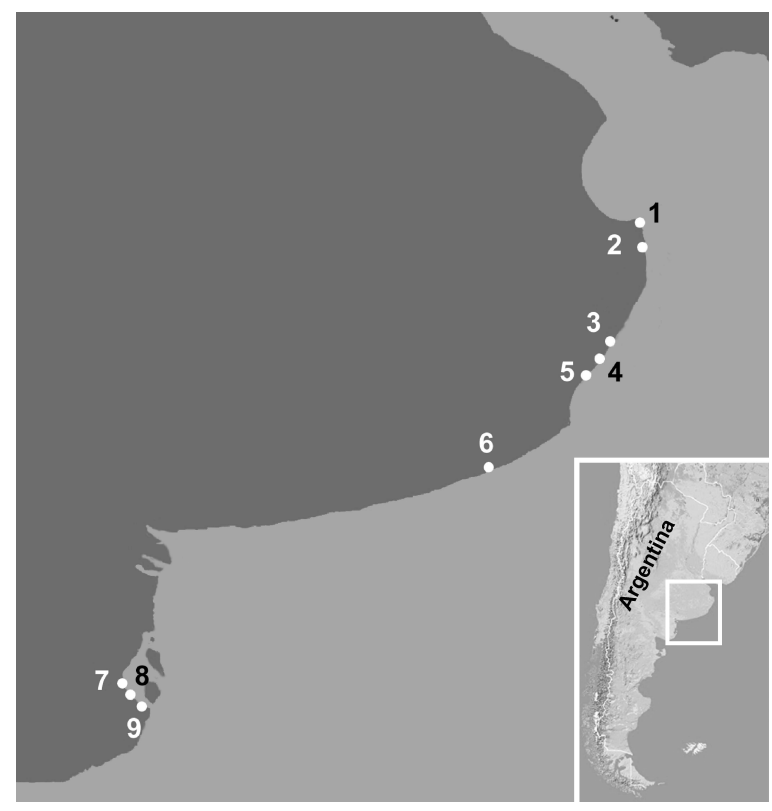

Fig. 1. Mapa del litoral marítimo de la Provincia de Buenos Aires mostrando las estaciones de muestreo y la localización del área en Argentina. 1: San Clemente del Tuyú; 2: Santa Teresita; 3: Pinamar; 4: Villa Gesell; 5: Mar Azul; 6: Puerto Quequén; 7: AR-BA 001 Los Pocitos; 8: AR-BA 002 Ría del Jabalí; 9: AR-BA 003 Bahía San Blas. 


\section{RESULTADOS}

\section{Toxinas PSP en la Zona Norte}

Previamente al comienzo formal del proyecto de monitoreo se obtuvieron bioensayos positivos para PSP en moluscos procedentes de Mar Azul, (concentración de toxina superior al límite máximo aceptable para el consumo humano, $80 \mu \mathrm{g}$ de STX equivalente por $100 \mathrm{~g}$ de carne de molusco) por lo que el 28-02-08 fue impuesta una veda al consumo y extracción de moluscos (Tabla 1). La especie asociada a la toxicidad de los moluscos fue Gymnodinium catenatum (Fig. 2), que apareció en concentraciones de $10^{3}$ a $10^{4}$ cél $1^{-1}$ (no graficado). En las muestras de fitoplancton obtenidas en la campaña del 14-03-08 aún la especie se encontraba en concentraciones de $7,14 \times 10^{3}$ cél $1^{-1}$ en San

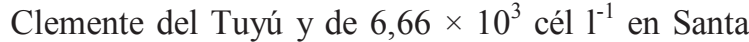
Teresita, y los bioensayos realizados sobre moluscos para toxinas PSP resultaron: positivo en Brachidontes rodriguezii y negativo en Mesodesma mactroides. Para la campaña del 24-04-08 $G$. catenatum se encontró sólo en las muestras cualitativas y los bioensayos fueron negativos para PSP en Mesodesma mactroides. La veda fue levantada el 05-05-08 (Tabla 1) luego de dos muestreos consecutivos en que no se hallaron concentraciones detectables de toxinas PSP en los moluscos.

Las muestras de Brachidontes rodriguezii procedentes de Santa Teresita obtenidas en la campaña del 15-02-11 dieron bioensayos positivos para PSP, por lo que el 24-02-11 fue impuesta una veda al consumo y extracción de moluscos (Tabla 1). En esta oportunidad la especie asociada a la toxicidad de los moluscos también fue Gymnodinium catenatum, presente en concentraciones de $1,67 \times 10^{4}$ cél $1^{-1}$ en San Clemente del Tuyú (Fig. 3). Para la campaña del 2502-11 la especie fue encontrada en las cuatro estaciones y presentó concentraciones que oscilaron

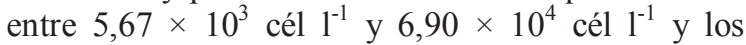
niveles de PSP superaron el límite sanitario en Mesodesma mactroides y en Brachidontes rodriguezii procedentes de San Clemente del Tuyú, Santa Teresita y Mar Azul. La veda fue levantada en fecha 18-05-11 (Tabla 1) luego de dos muestreos consecutivos en que los moluscos resultaron aptos para consumo humano. Sin embargo quedó en vigencia una veda por toxinas lipofílicas porque los bioensayos para lipofílicas fueron positivos (Fig. 3).
El siguiente brote de toxinas PSP en moluscos fue registrado mediante bioensayo en ratón realizado sobre muestras de Brachidontes rodriguezii colectadas en la campaña del 23-02-12 en Santa Teresita, por lo que en fecha 29-02-12 fue impuesta una veda al consumo y extracción de moluscos (Tabla 1). Como en las oportunidades anteriores la especie asociada a la toxicidad en los moluscos fue Gymnodinium catenatum, presente en

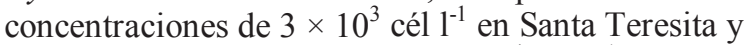

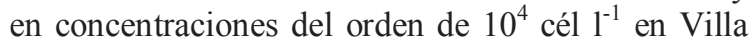
Gesell y Mar Azul (Fig. 3). Esta veda fue dejada sin efecto el 15-05-12 luego de que los niveles de PSP en moluscos fueron no detectables en todas las estaciones de muestreo (Tabla 1). Sin embargo quedó en vigencia la veda anteriormente impuesta luego de obtener bioensayos positivos para toxinas lipofílicas porque los moluscos seguían estando contaminados con estas toxinas (Fig. 3).

Durante todo el período de muestreo no hemos detectado ningún episodio de toxicidad por PSP asociado a Alexandrium tamarense en las estaciones comprendidas entre San Clemente del Tuyú y Mar Azul, y sólo en los muestreos del 27-09-10 y del 1001-11 la especie estuvo presente en concentraciones del orden de $10^{3}$ cél $1^{-1}$.

Las muestras de Brachidontes rodriguezii colectadas en Puerto Quequén durante la campaña del 21-09-09 resultaron positivas para PSP por lo que el 25-09-09 fue impuesta una veda al consumo y extracción de moluscos (Tabla 1). La especie asociada a la toxicidad de los moluscos fue Alexandrium tamarense, que apareció en concentraciones de $8,3 \times 10^{3}$ cél $1^{1}$ y no fue hallada en las muestras cuantitativas en el resto de las estaciones de muestreo. La veda fue levantada el 1812-09 (Tabla 1) luego de dos muestreos consecutivos en que se obtuvieron bioensayos negativos de los moluscos.

\section{Toxinas PSP en la Zona Sur}

En esta zona Alexandrium tamarense sólo fue encontrada durante la campaña del 01-09-09 en concentraciones de $1,0 \times 10^{3}$ cél $1^{-1}$ en AR-BA 002

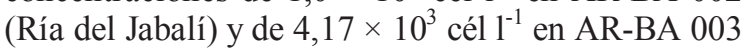
(Bahía San Blas), sin que fueran detectadas toxinas PSP en Crassostrea gigas, y Gymnodinium catenatum no fue hallada en las muestras cuantitativas en ninguno de los muestreos llevados a cabo a lo largo del período. 
Inés Sunesen et al. - Episodios de toxicidad en moluscos asociados a algas toxígenas

Tabla 1. Resumen de las vedas y alertas sanitarias impuestas por la autoridad de aplicación entre marzo de 2008-marzo de 2013, fechas de inicio y finalización con sus resoluciones o disposiciones, toxina encontrada en cada caso y duración de cada veda. SC = San Clemente del Tuyú, ST = Santa Teresita, VG = Villa Gesell, MAz = Mar Azul; *, estación de muestreo eventual.

\begin{tabular}{|c|c|c|c|c|c|}
\hline Zona & Estaciones & $\begin{array}{l}\text { Fecha de inicio de la } \\
\text { veda }\end{array}$ & $\begin{array}{c}\text { Fecha de } \\
\text { finalización de la veda }\end{array}$ & Toxinas & $\begin{array}{c}\text { Días } \\
\text { totales de } \\
\text { veda }\end{array}$ \\
\hline \multirow[t]{7}{*}{ Zona Norte } & SC,ST,VG,MAz & 28-02-08 (R 02/08) & 05-05-08 (R 09/08) & PSP & 38 \\
\hline & *Quequén & *25-09-09 (R 07/09) & 18-12-09 (R 98/09) & PSP & 84 \\
\hline & SC,ST,VG,MAz & 03-02-10 (D 13/10) & 13-07-11 (D 33/11) & DSP & 528 \\
\hline & SC,ST,VG,MAz & 24-02-11 (D 17/11) & 18-05-11(D 24/11) & PSP & 84 \\
\hline & SC,ST,VG,MAz & $24-02-12$ (R 17/12) & 30-07-12 (R 131/12) & DSP & 158 \\
\hline & SC,ST,VG,MAz & 29-02-12 (R23/12) & $15-05-12$ (R 87/12) & PSP & 77 \\
\hline & SC,ST,VG,MAz & $10-12-12$ (D 08/12) & vigente & DSP & \\
\hline \multirow[t]{11}{*}{ Zona Sur } & AR-BA 001 & 23-12-10 alerta (D 88/10) & & lipofílicas & \\
\hline & Los Pocitos & $10-02-11$ (D 14/11) & 06-05-11 (D 23/11) & lipofílicas & 135 \\
\hline & & 01-10-12 alerta (D 04/12) & $16-10-12$ (D 05/12) & lipofílicas & 15 \\
\hline & & $12-03-13 \mathrm{D}(18 / 13)$ & $11-04-13$ (D 22/13) & lipofílicas & 30 \\
\hline & AR-BA 002 & 03-02-09 (D 05/09) & $18-02-09$ (D 06/09) & lipofílicas & 15 \\
\hline & Ría del Jabalí & 23-12-10 alerta (D 88/10) & & lipofílicas & \\
\hline & & $10-02-11$ (D 14/11) & 20-04-11 (D 22/11) & lipofílicas & 119 \\
\hline & AR-BA 003 & 03-02-09 (D 05/09) & $18-02-09$ (D 06/09) & lipofílicas & 15 \\
\hline & Bahía San Blas & 23-12-10 alerta (D 88/10) & & lipofílicas & \\
\hline & & $10-02-11$ (D 14/11) & 20-04-11 (D 22/11) & lipofílicas & 119 \\
\hline & & $12-09-11$ (D40/11) & 30-09-11 (D 45/11) & lipofílicas & 18 \\
\hline
\end{tabular}

Toxinas lipofilicas en la Zona Norte

En las muestras de fitoplancton obtenidas durante la campaña del 16-11-09 fue detectada Dinophysis acuminata alcanzando concentraciones

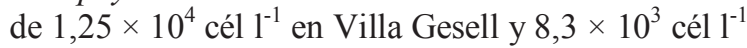
en Mar Azul. Para enero de 2010 se obtuvieron bioensayos positivos para toxinas lipofílicas en moluscos de Villa Gesell y Mar Azul por lo que a partir del día 03-02-10 fue impuesta una veda total para la extracción comercial, artesanal y/o turística de moluscos bivalvos (Tabla 1). Estas toxinas no habían sido detectadas para la Provincia de Buenos Aires previamente a la ejecución de este proyecto de monitoreo y consistentemente con su hallazgo en moluscos contaminados fue encontrada Dinophysis acuminata (Fig. 4) en las muestras de fitoplancton obtenidas durante la campaña del 04-01-10

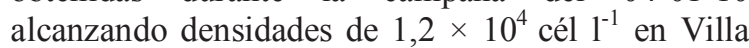

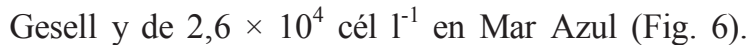
Para la campaña del 28-01-10 en las mismas estaciones, además de hallarse concentraciones del orden de $10^{4}$ cél $1^{-1}$ de Dinophysis acuminata se encontraron concentraciones del orden de $10^{3}$ cél $1^{-1}$ de otra especie toxígena, D. caudata (Fig. 5). Simultáneamente con el hallazgo del brote de toxicidad fueron reportados ante el Departamento de Bromatología de Pinamar (Prov. de Buenos Aires) nueve casos de pacientes con gastroenteritis afebril asociada a la ingesta de berberechos cocidos (Donax hanleyanus) colectados en Villa Gesell. Los moluscos cocidos resultaron positivos para toxinas lipofílicas por bioensayo en ratón y los signos presentados por los animales experimentales, así como los síntomas de los intoxicados, fueron consistentes con los de la intoxicación diarreica por 
consumo de moluscos (DSP) (Sar et al., 2010; Goya et al., 2012). Las muestras de moluscos fueron ulteriormente analizadas por HPLC-FLD confirmándose la presencia de Ácido Okadaico (AO), Dinophysistoxina-1 (DTX-1) y los Acylderivados de éstos: Acyl-Ácido Okadaico (AcylOA) y Acyl-Dinophysistoxina-1 (Acyl-DTX-1) (Sar et al., 2012). La veda se prolongó por espacio de un año y medio hasta el 13-07-11 en que fue dejada sin efecto luego de que se obtuviera el segundo resultado negativo para toxinas lipofílicas en moluscos colectados el 27-06-11. Durante ese largo período Dinophysis acuminata presentó concentraciones fluctuantes en el fitoplancton, llegando a desaparecer de las muestras cuantitativas de un mes y alcanzando concentraciones del orden de $10^{2}$ y hasta $10^{4}$ cél $1^{-1}$ en el siguiente. En las muestras colectadas en la campaña del 10-01-11, y antes de que los moluscos estuvieran aptos para el consumo humano, Dinophysis acuminata y $D$. caudata aparecieron nuevamente juntas en Mar Azul

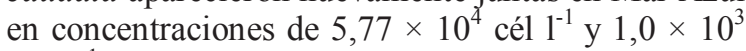
cél $1^{-1}$ respectivamente y $D$. acuminata alcanzó concentraciones del orden de $10^{4}$ cél $1^{-1}$ en Villa Gesell, y de $10^{3}$ cél $1^{-1}$ en San Clemente del Tuyú y Santa Teresita. Esta veda se superpuso parcialmente con la veda establecida el 24-02-11 a causa de un brote de toxinas PSP.

En enero de 2012 se obtuvieron bioensayos positivos para DSP en Brachidontes rodriguezii colectados en la campaña del 28-01-12 en un solo punto de la Zona Norte, Pinamar, por lo que a partir del 24-02-12 fue impuesta una veda a la extracción de moluscos (Tabla 1). La especie asociada a la toxicidad en los moluscos fue $D$. acuminata que al momento de la recolección de las muestras se presentó en tan baja concentración que sólo pudo ser observada en la muestra cualitativa. Las concentraciones de D. acuminata llegaron durante el período de veda a ser del orden de $10^{2}$ en San Clemente del Tuyú, Villa Gesell y Mar Azul y de

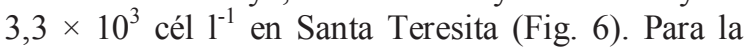
campaña del 11-06-12 se obtuvo el segundo bioensayo negativo para DSP en Mesodesma mactroides y la veda fue dejada sin efecto el 30-0712.

Otro brote de toxinas DSP fue detectado por bioensayo en material de Brachidontes rodriguezii procedente de Santa Teresita colectado durante la campaña del 21-11-12, esto dio lugar a la imposición de una veda el 10-12-12. La especie asociada a este episodio de toxicidad en moluscos fue Dinophysis caudata que fue hallada en una concentración de 3,3

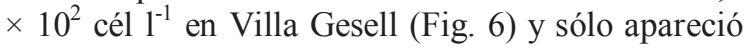
en las muestras cualitativas en Santa Teresita. En las muestras colectadas durante la campaña del 27-1212 D. caudata se presentó en concentraciones de 6,2

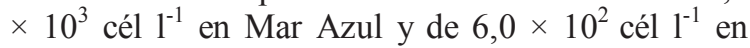
Villa Gesell, y $D$. acuminata y $D$. tripos se encontraron en concentraciones de $10^{2}$ cél $1^{-1}$ en Mar
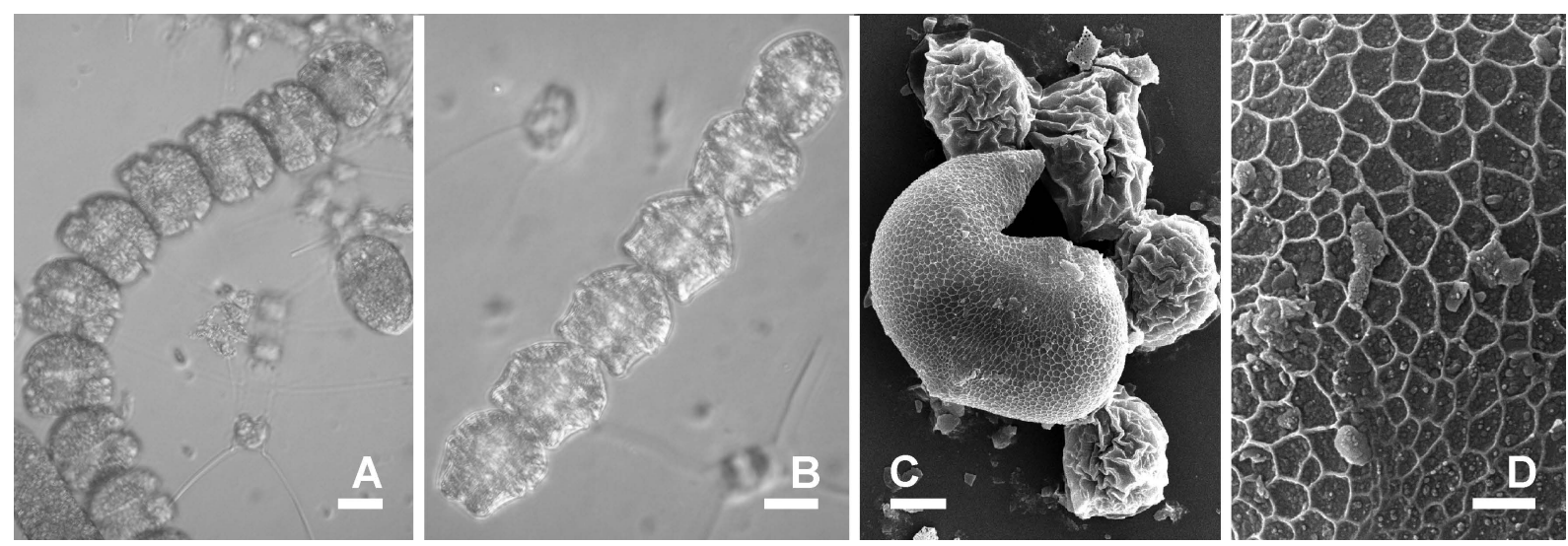

Fig. 2. Gymnodinium catenatum. MO. A-B: Aspecto general de la colonia. MEB. C: Quiste mostrando el arqueópilo, abertura de exquistamiento formada durante la fase de germinación. D: Detalle de la pared reticulada del quiste. Escalas: $A-B=20 \mu \mathrm{m}, C=10 \mu \mathrm{m}, \mathrm{D}=2 \mu \mathrm{m}$ 
Inés Sunesen et al. - Episodios de toxicidad en moluscos asociados a algas toxígenas
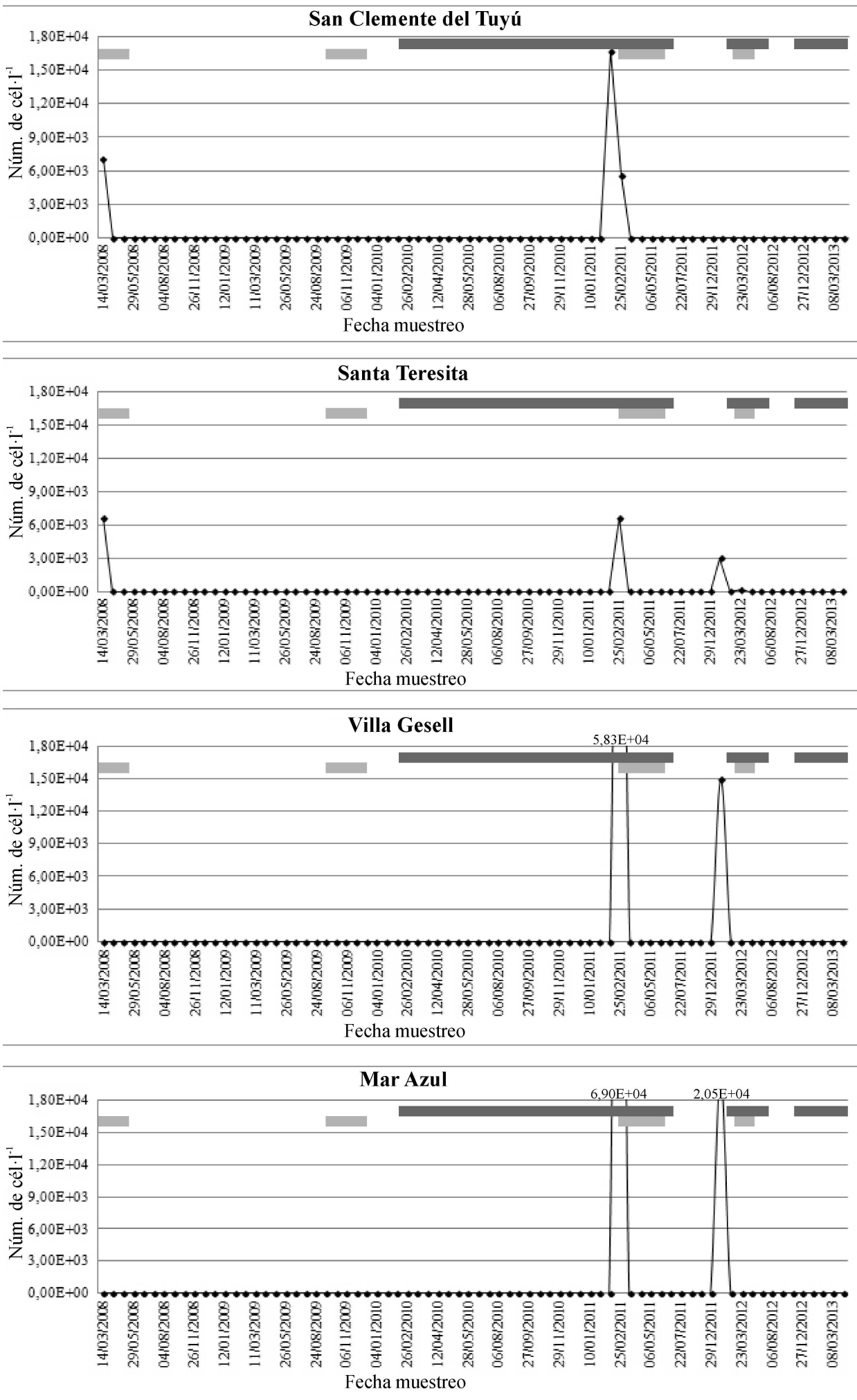

Fig. 3. Distribución temporal y densidad de Gymnodinium catenatum en la Zona Norte.

- Períodos de vedas por PSP. — Períodos de vedas por DSP. 
Azul y sólo en las muestras cualitativas en Santa Teresita, sin embargo los moluscos procedentes de Mar Azul y Santa Teresita (Donax hanleyanus y Mesodesma mactroides respectivamente) dieron bioensayos positivos para DSP. Para la campaña del 10-01-13 las especies de Dinophysis sólo aparecieron en las muestras cualitativas y para la siguiente del 29-01-13 alcanzaron concentraciones

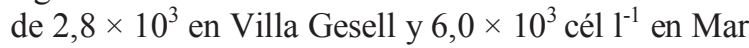
Azul. Para el mes de marzo de 2013 al finalizar el período bajo análisis la veda aún no había sido levantada.

\section{Toxinas lipofilicas en la Zona Sur}

En enero de 2009 fue detectado un brote de toxinas lipofílicas mediante bioensayo ratón en Crassostrea gigas procedente de AR-BA 002 (Ría del Jabalí) y de AR-BA 003 (Bahía San Blas) por lo que el 03-02-09 fue impuesta una veda a la extracción y consumo de moluscos (Tabla 1). En las muestras de fitoplancton no fueron halladas especies que pudieran ser asociadas a la producción de toxinas lipofílicas. La veda fue levantada en ambas zonas en fecha 18-02-09 (Tabla 1) luego de determinar mediante bioensayos ratón que los moluscos estaban aptos para consumo humano.

Los bioensayos para toxinas lipofílicas realizados sobre muestras de ostras colectadas el 2710-10 en AR-BA 001 (Los Pocitos), AR-BA 002 y AR-BA 003, arrojaron resultados "no concluyentes por interferencia de otras toxinas" (sic Informe de SENASA). Los profesionales de SENASA observaron que luego de la inoculación de los extractos lipofílicos los ratones presentaban un tiempo de supervivencia inusualmente corto, inferior a 5 minutos, y que la signología no era consistente con la descripta para toxinas lipofílicas. Coherentemente no fueron halladas especies productoras de DSP en los análisis de fitoplancton realizados rutinariamente en la zona para la fecha.
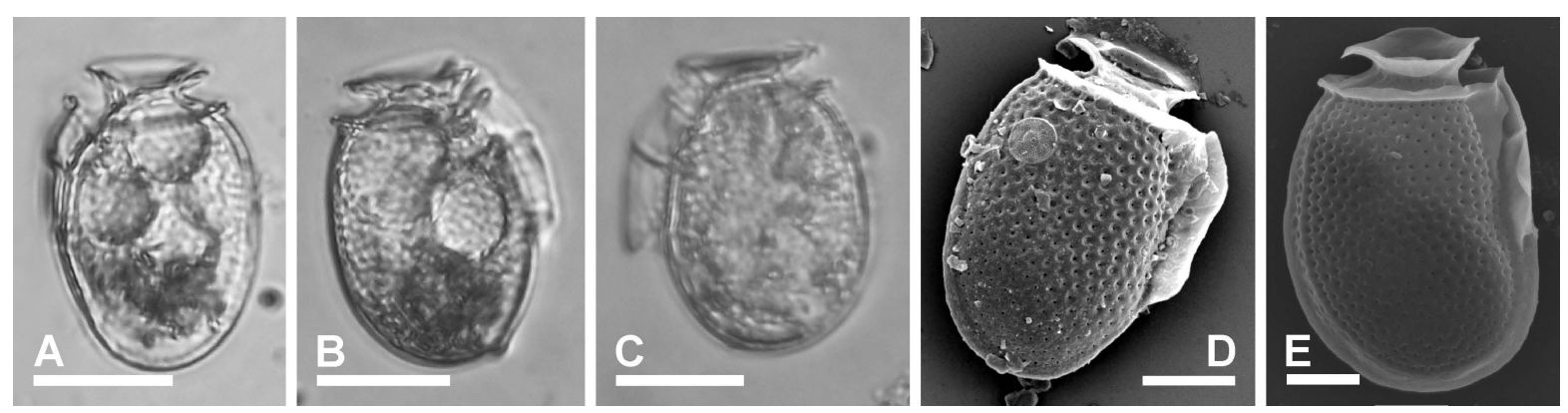

Fig. 4. Dinophysis acuminata. MO. A-C: Células en vista lateral. Note la variabilidad morfológica de la especie. MEB. D-E: Células en vista lateral derecha. Note que el ala sulcal derecha sobrepasa la segunda costilla del ala sulcal izquierda. Escalas: $A-C=20 \mu \mathrm{m}, \mathrm{D}-\mathrm{E}=10 \mu \mathrm{m}$.
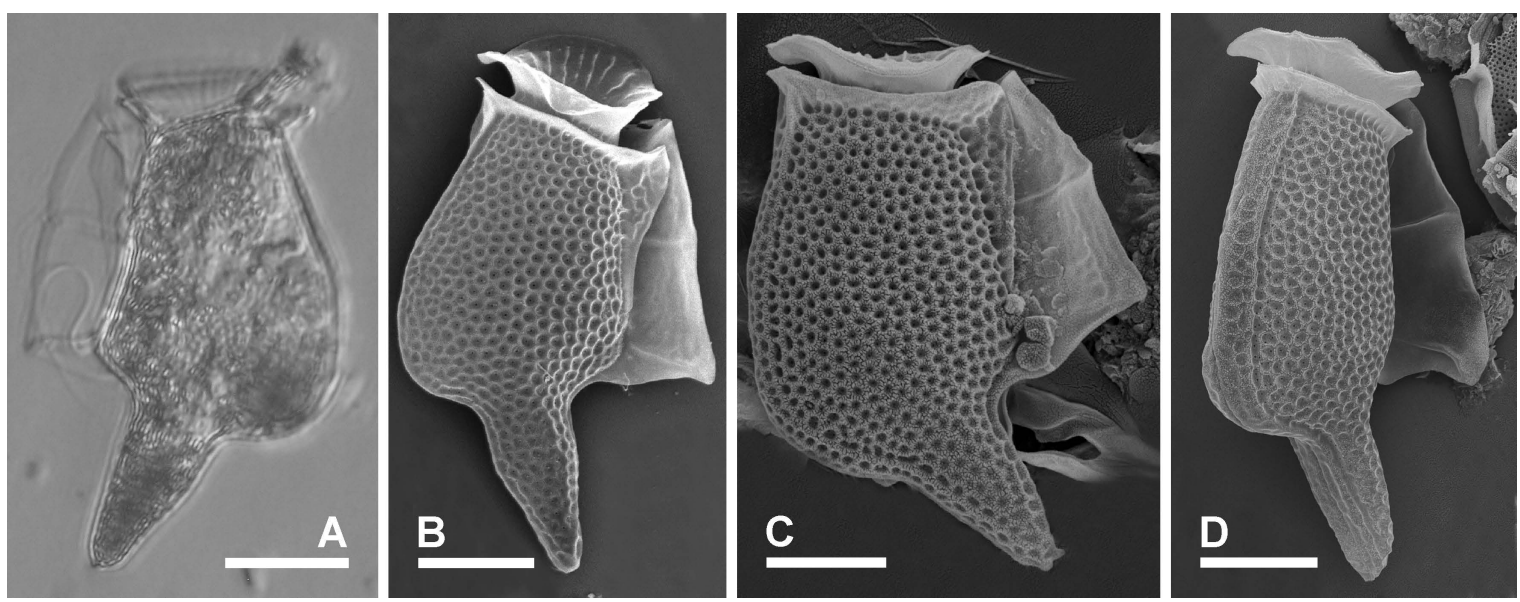

Fig. 5. Dinophysis caudata. MO. A: Célula en vista lateral. MEB. B-C: Células en vista lateral derecha. Note la variabilidad morfológica de la especie. D: Célula en vista lateral inclinada mostrando la sutura dorsiventral. Escalas: $A-D=20 \mu \mathrm{m}$. 


\section{Inés Sunesen et al. - Episodios de toxicidad en moluscos asociados a algas toxígenas}

Sobre la base de los resultados emitidos por el SENASA, la autoridad de aplicación resolvió establecer una alerta sanitaria en fecha 23-12-10 (Tabla 1), a los fines de resguardar la salud pública. A partir de enero de 2011, se informaron resultados positivos para toxinas lipofílicas en las muestras de ostras procedentes de AR-BA 001, AR-BA 002 y AR-BA 003, por lo que la autoridad de aplicación resolvió establecer una veda total a la extracción, consumo y comercialización a partir del 10-02-11. Esta veda concluyó el 20-04-11 para AR-BA 002 y AR-BA 003 y el 06-05-11 para AR-BA 001 (Tabla 1) luego de obtenerse resultados negativos para toxinas lipofilicas durante dos campañas consecutivas. Los bioensayos negativos que dieron lugar al levantamiento de la veda tuvieron también particularidades, los ratones inoculados entraban en shock por 30 a 60 minutos y luego se recobraban.

Los análisis de contra-muestras por LC-MS/MS llevados a cabo posteriormente en el Laboratorio de Referencia Comunitario (U.E.) para Biotoxinas Marinas (Vigo, España), resultaron negativos para azaspirácidos, ácido okadaico, dinofisistoxinas, pectenotoxinas y yesotoxinas (toxinas lipofilicas reguladas) y para espirólidos (toxina lipofílica no regulada).

Un episodio de similares características sucedió a partir de agosto de 2011, nuevamente SENASA obtuvo resultado no concluyente en el bioensayo para toxinas lipofílicas en Crassostrea gigas procedente de AR-BA 003, señalando muerte atípica fulminante de los ratones luego de 5 minutos de inoculados. Sobre esta base la autoridad de aplicación resolvió establecer una alerta sanitaria a partir del 12-09-11 mediante la cual indicó a la población que se abstuviera de extraer, comercializar y consumir ostra procedente de Bahía San Blas (Tabla 1). En fecha 30-09-11 fue levantada la alerta luego de determinar que los moluscos estaban aptos para consumo humano (Tabla 1).

Otro brote de toxinas lipofílicas fue detectado mediante bioensayo en ratón en material de Crassostrea gigas procedente de AR-BA 001, colectado durante la campaña del 17-09-12, por lo que fue impuesta una veda a la extracción, consumo y comercialización de moluscos a partir del 01-1012 (Tabla 1). Las muestras de fitoplancton colectadas en la campaña correspondiente no presentaron especies que pudieran ser asociadas a la producción de toxinas lipofílicas. La veda fue levantada en fecha 16-10-12 luego de determinar que los moluscos estaban aptos para consumo humano (Tabla 1).
En marzo de 2013 se obtuvo bioensayo positivo para toxinas lipofilicas en Crassostrea gigas procedente de AR-BA 001, por lo que fue impuesta una veda a partir del 12-03-13 (Tabla 1). Como en los casos previos no se detectaron especies en las muestras correspondientes de fitoplancton que pudieran ser asociadas a la producción de estas toxinas. La veda fue levantada en fecha 11-04-13 (Tabla 1) luego de obtener dos resultados negativos consecutivos.

\section{Discusión}

Durante el período monitoreado fueron detectados tres brotes de toxinas PSP en moluscos de la Zona Norte de la Provincia de Buenos Aires asociados a la presencia de Gymnodinium catenatum en el fitoplancton, todos ellos tuvieron su inicio hacia finales de febrero de los años 2008, 2011 y 2012, durante el verano austral. Este patrón de aparición de la especie y del brote de toxicidad en moluscos muestra semejanzas con el descripto por Méndez \& Ferrari (2002) para aguas costeras de Uruguay durante el período 1992-1998, sin embargo están más acotados en el tiempo en la Provincia de Buenos Aires que en la costa uruguaya. Akselman et al. (1998) realizaron un análisis de niveles de toxicidad por PSP en bancos de Mytilus edulis Linnaeus (mejillón) de Mar del Plata y detectaron picos otoñales de toxicidad (entre marzo y mayo) para los años 1984, 1990, 1992 y 1993 que asociaron a la aparición de G. catenatum en el fitoplancton del área, en baja abundancia, durante el otoño. En este estudio en cambio la aparición de esta especie en el fitoplancton en concentraciones del orden de $10^{3}$ a $10^{4}$ cél $1^{-1}$ y los picos de toxicidad en moluscos fueron más tempranos y más acotados en el tiempo que los descriptos por Akselman et al. (1998).

En la Zona Sur no fueron detectados brotes de toxinas PSP durante el período bajo estudio, concordantemente Gymnodinium catenatum no fue hallada en ninguna de las muestras cuantitativas a lo largo del período analizado y Alexandrium tamarense sólo fue encontrada en las muestras cuantitativas de la campaña 01-09-09 en concentraciones del orden de $10^{3}$ cél $1^{-1}$ para AR-BA 002 y AR-BA 003.

El único episodio de toxinas PSP en moluscos asociado a la proliferación de Alexandrium tamarense en el fitoplancton, fue detectado en 
Bol. Soc. Argent. Bot. 49 (3) 2014
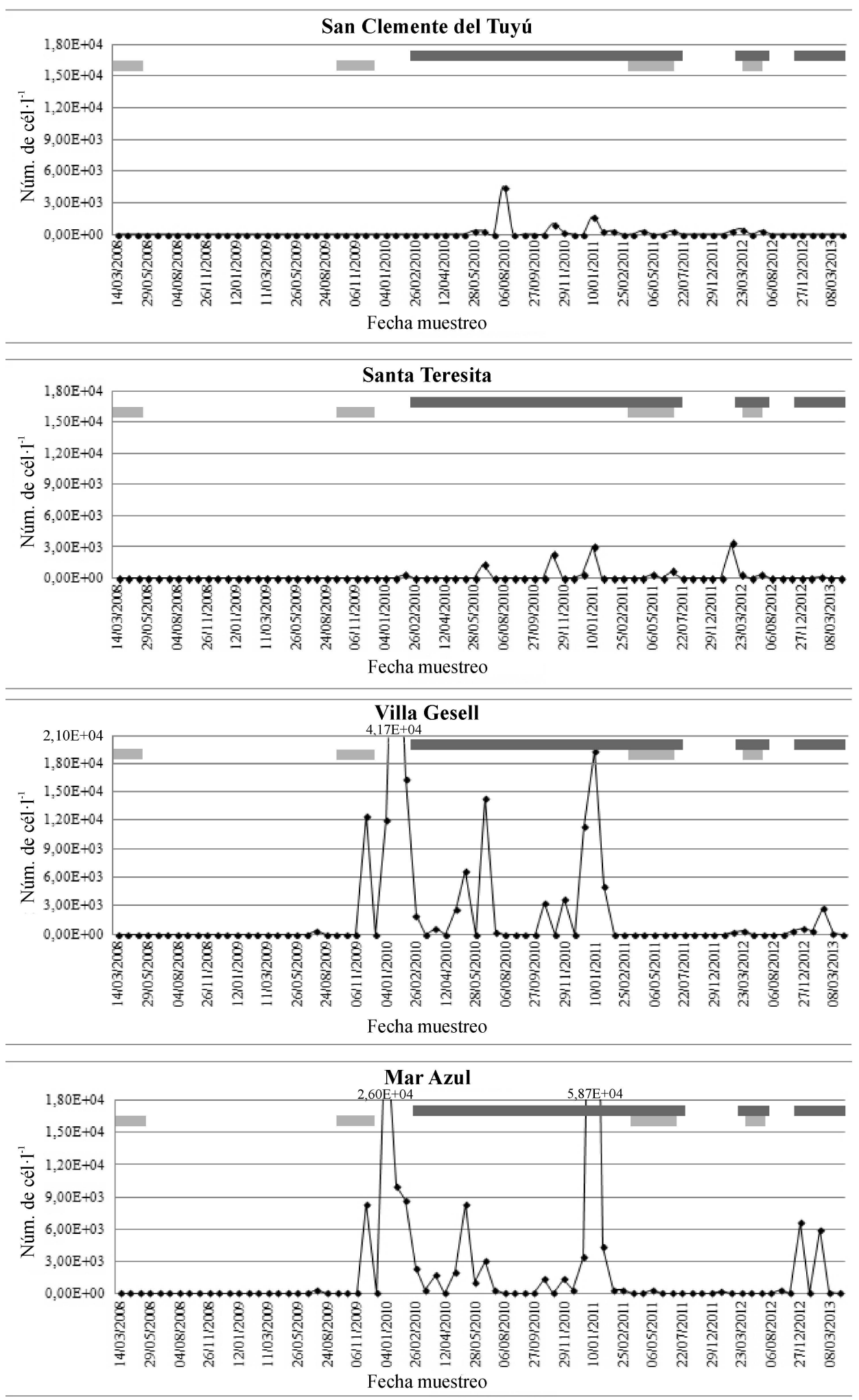

Fig. 6. Distribución temporal y densidad de Dinophysis acuminata + D. caudata en la Zona Norte.

- Períodos de vedas por PSP. " Períodos de vedas por DSP 
Puerto Quequén, fuera de las zonas regularmente monitoreadas, y se produjo en la primavera en concordancia con el patrón previamente descripto por Carreto et al. (1996, 1998 a y b).

Los tres brotes de toxinas DSP en la Zona Norte asociados a Dinophysis acuminata y D. caudata se iniciaron entre la primavera tardía y el verano temprano, patrón que muestra semejanzas con el descripto por Méndez \& Ferrari (2002) para las aguas costeras de Uruguay.

Gayoso \& Ciocco (2001) y Gayoso et al. (2002) reportaron el primer brote de toxinas DSP en Argentina en asociación con la presencia del dinoflagelado bentónico Prorocentrum lima (Ehrenberg) Dodge y el primer episodio de intoxicación diarreica por consumo de moluscos en humanos en el Golfo Nuevo, Provincia de Chubut. A partir de este monitoreo fue realizado el primer reporte para Argentina de acumulación de toxinas DSP en moluscos vinculada a la aparición de Dinophysis acuminata y D. caudata en el plancton (Sar et al., 2010), el primer hallazgo de Acylderivados para el Atlántico Sudamericano, el primer hallazgo de OA para Argentina (Sar et al., 2012) y el primer reporte de intoxicación alimentaria por DSP para la Provincia de Buenos Aires (Goya et al., 2012).

Las toxinas lipofílicas detectadas en muestras de ostras procedentes de la Zona Sur que produjeron brevísimos tiempos de supervivencia de los animales experimentales podrían ser iminas cíclicas, a las que se denomina "fast acting toxins" (Aune, 2008; Cembella \& Krock, 2008). Estas toxinas están caracterizadas porque al ser inoculadas provocan mortalidad a los ratones en corto tiempo, con signos característicos de PSP (Fernández et al., 2002, Tabla 3: 92), cuando la concentración es letal y shock inicial seguido de restablecimiento completo cuando la concentración es sub-letal (EFSA, 2010). Según la opinión científica emitida por la European Food Safety Authority (EFSA, 2010), no hay límite regulatorio establecido en Europa y en otras regiones del mundo para ninguna de las iminas cíclicas. Ante la repetición de eventos de toxicidad con las mismas características, nuestro grupo de trabajo ha propuesto al Director Provincial de Pesca hacer el envío de contra-muestras del primer episodio conservadas con propósito de investigación, a químicos especialistas en este tipo de toxinas.

Las vedas impuestas a la extracción, consumo y comercialización de moluscos en salvaguarda de la salud pública y las economías costeras, la causal de cada una de ellas y el tiempo por el que se mantuvieron vedados los recursos son resumidos en la Tabla 1.

\section{AgRADECIMIENTOS}

A Mariano González, actual Director Provincial de Pesca del Ministerio de Asuntos Agrarios de la Provincia de Buenos Aires, por su apoyo en lo atinente a la realización de los viajes de campaña. A Gabriel Meléndez, Coordinador General Regional del Centro Regional Buenos Aires Sur, Mar del Plata, del SENASA, por su apoyo técnico y asistencia económica. A Marcela Alvarez, de la Dirección de Acuicultura del Ministerio de Agricultura, Ganadería y Pesca de la Nación, por la organización del Taller "Biotoxinas marinas y floraciones algales nocivas", primera iniciativa a nivel nacional de coordinar diferentes sectores vinculados a la problemática. Y al Sr. Christian Maurs, pescador artesanal, por su colaboración desinteresada en la obtención de muestras de moluscos. La investigación fue financiada con subsidios de la Universidad Nacional de La Plata N/11/640, del Consejo Nacional de Investigaciones Científicas y Técnicas PIP0067 y del Consejo Federal Pesquero.

\section{BibLIOGRAFÍA}

AKSELMAN, R., J. I. CARRETO \& N. G. MONTOYA. 1998. Gymnodinium catenatum and autumn toxicity in Northern shelf waters of Argentina. In: REGUERA, B., BLANCO J., M. L. FERNÁNDEZ \& T. WYATT (eds.), Harmful Algae, pp. 122-123. Xunta de Galicia and IOC UNESCO, Santiago de Compostela.

AOAC. 1995. Paralytic shellfish poison. Biological method. Final action. In: Official Methods of Analysis, 35: 21-22. Association of Official Analytical Chemists, Gaithersburg. [sec 959.08]

AUNE, T. 2008. Risk assessment of marine toxins. In: Botana, L. M. (ed.), Seafood and freshwater toxins, pp. 3-20. CRC Press, Taylor \& Francis, Boca Raton.

BALECH, E. 1995. The genus Alexandrium Halim (Dinoflagellata). Sherkin Island Marine Station, Sherkin Island. 
CARRETO, J. I. \& R. AKSELMAN. 1996. Gymnodinium catenatum and autumnal toxicity in Mar del Plata. Harmful Algae News 15: 1-3.

CARRETO, J. I., R. AKSELMAN, N. G. MONTOYA, R. M. NEGRI, H. R. BENAVIDES, M. O. CARIGNAN \& A. D. CUCCHI COLLEONI. 1998a. Alexandrium tamarense bloom dynamics and Mytilus edulis toxicity in the coastal waters off Mar del Plata (Argentina). In: REGUERA, B., J. BLANCO, M. L. FERNÁNDEZ \& T. WYATT (eds.), Harmful Microalgae, pp. 135-138. IOCUNESCO, Santiago de Compostela.

CARRETO, J. I., C. ElBUSTO, H. SANCHO, M. O. CARIGNAN, T. YASUMOTO \& Y. OSHIMA. 1996. Comparative studies on paralytic shellfish toxin profiles of marine snails, mussels and an Alexandrium tamarense isolate from the Mar del Plata coast (Argentina). Rev. Invest. Desarr. Pesq. 10: 101-107.

CARRETO, J. I., M. LASTA, R. M. NEGRI \& H. R. BENAVIDES. 1981. Los fenómenos de marea roja y toxicidad de moluscos bivalvos en el Mar Argentino. Contr. INIDEP 399: 1-55.

CARRETO, J. I., N. G. MONTOYA, A. D. CUCCHI COLLEONI \& R. AKSELMAN. 1998b. Alexandrium tamarense blooms and shellfish toxicity in the Argentine Sea: a retrospective view. In: REGUERA, B., J. BLANCO, M. L. FERNÁNDEZ \& T. WYATT (eds.), Harmful Microalgae, pp. 131-134. IOCUNESCO, Santiago de Compostela.

CEMBELLA, A. \& B. KROCK. 2008. Cyclic imine toxins: chemistry, biogeography, biosynthesis and pharmacology. In: BOTANA, L. M. (ed), Seafood and freshwater toxins, pp. 561-580. CRC Press, Taylor \& Francis, Boca Raton.

EFSA (EUROPEAN FOOD SAFETY AUTHORITY). 2010. Scientific opinion on marine biotoxins in shellfish - Cyclic imines (spirolides, gymnodimines, pinnatoxins and pteriatoxins). EFSA Journal 8: 1628.

FERNÁNDEZ, M. L., A. MÍGUEZ, E. CACHO, A. MARTÍNEZ, J. DIOGËNE \& $\mathrm{T}$. YASUMOTO. 2002. Bioensayos con mamíferos y ensayos bioquímicos y celulares para la detección de ficotoxinas. In: SAR, E. A., M. E. FERRARIO \& B. REGUERA (eds.), Floraciones algales nocivas en el Cono Sur Americano, pp. 77-120. Instituto Español de Oceanografía, Madrid.
GARCÍA, C., M. PRUZZO, N. RODRÍGUEZUNDA, C. CONTRERAS \& N. LAGOS. 2010. First evidence of Okadaic acid acyl-derivative and Dinophysistoxin-3 in mussel samples collected in Chiloe Island, Southern Chile. J. Toxicol. Sci. 35: 335-344.

GAYOSO, A. M. \& N. CIOCCO. 2001. Observations on Prorocentrum lima of NorthPatagonian coastal waters (Argentina) associated with a diarrhoeic disease episode. Harmful Algae News 22: 4.

GAYOSO, A. M., S. DOVER, S. L. MORTON, M. BUSMAN, P. D. R. MOELLER \& L. MARANDA. 2002. Possibility of diarrheic shellfish poisoning associated with Prorocentrum lima (Dinophyceae) in Patagonian Gulfs (Argentina). J. Shellfish Res. 21: 461-463.

GOYA, A, E. SANGORRÍN, A. LAVIGNE, I. SUNESEN, S. LOFEUDO \& E. A. SAR. 2012. Toxinas Diarreicas de los Moluscos, Primer reporte de intoxicación alimentaria en la Provincia de Buenos Aires. Revista del Colegio de Veterinarios de la Provincia de Buenos Aires, Suplemento Técnico Veterinario 50: 4849.

MÉNDEZ, S. \& G. FERRARI. 2002. Floraciones algales nocivas en Uruguay: Antecedentes, proyectos en curso y revisión de resultados. In: SAR, E. A., M. E. FERRARIO \& B. REGUERA (eds.), Floraciones algales nocivas en el Cono Sur Americano, pp. 269-288. Instituto Español de Oceanografía, Madrid.

NEGRI, R. M., N. MONTOYA, J. I. CARRETO, R. AKSELMAN \& D. INZA. 2004. Pseudonitzschia australis, Mytilus edulis, Engraulis anchoita and domoic acid in the Argentine Sea. In: STEIDINGER, K. A., J. H. LANDSBERG, C. R. TOMAS \& G. A. VARGO (eds.), Proceedings Xth Conference, Harmful Algal Blooms 2002, pp. 139-141. Florida Fish and Wildlife Conservation Commission, Florida Institute of Oceanography and Intergovernmental Oceanographic Commission of UNESCO, St Petersburg.

REGUERA, B. 2002. Establecimiento de un programa de seguimiento de microalgas tóxicas. In: SAR, E. A., M. E. FERRARIO \& B. REGUERA (eds.), Floraciones algales nocivas en el Cono Sur Americano, pp. 19-54. Instituto Español de Oceanografia, Madrid. 
SAR, E. A., I. SUNESEN, A. B. GOYA, A. S. LAVIGNE, C. GARCÍA \& N. LAGOS. 2012. First report of Diarrhetic Shellfish Toxins in mollusks from Buenos Aires Province (Argentina) associated to Dinophysis spp.: Evidence of Okadaic acid, Dinophysistoxin-1 and Acyl-derivatives. Bol. Soc. Argent. Bot. 47: 5-14.

SAR, E. A., I. SUNESEN, A. S. LAVIGNE \& A. B. GOYA. 2010. Dinophysis spp. asociadas a detección de toxinas diarreicas en moluscos (DSTs) y aintoxicación diarreica en humanos (Provincia de Buenos Aires, Argentina). Rev. Biol. Mar. Oceanogr. 45: 451-460.
YASUMOTO, T., M. MURATA, Y. OSHIMA, K. MATSUMOTO \& J. CLARDY. 1984. Diarrhetic Shellfish Poisoning. In: RAGELIS E. (ed.), Seafood Toxins. ACS Symposium series 262, pp. 207-214. American Chemical Society, Washington D. C.

Recibido el 28 de noviembre de 2013, aceptado el 12 de marzo de 2014 